\title{
DID EGRET DETECT DISTANT SUPERNOVA REMNANTS?
}

\author{
Diego F. Torres ${ }^{1}$, Gustavo E. Romero ${ }^{2}$, Thomas M. Dame ${ }^{3}$, Jorge A. Combi ${ }^{3}$, Yousaf M. Butt ${ }^{4}$ \\ ${ }^{1}$ Lawrence Livermore Laboratory, 7000 East Ave. L-413, Livermore, CA 94550, USA \\ ${ }^{2}$ Instituto Argentino de Radioastronomía, C.C.5, 1894 Villa Elisa, Buenos Aires, Argentina \\ ${ }^{3}$ Harvard-Smithsonian Center for Astrophysics, 60 Garden Street, Cambridge, MA 02138, USA
}

\begin{abstract}
It might be thought that supernova remnants (SNRs) more distant than a few kiloparsec from Earth could not have been detected by the EGRET experiment. This work analyzes the observational status of this statement in the light of new CO studies of SNRs.
\end{abstract}

\section{INTRODUCTION}

When analyzing the $\gamma$-ray detections in the COS-B and partial EGRET catalogs, it was commonly believed that SNRs more distant than a few kpc from Earth could not have been detected by these experiments. The inverse square dependence of the flux on the distance to the source was, of course, critical in reaching such a conclusion. However, it is not only the distance but also the molecular environment that is crucial in determining detectability. In this presentation we analyze, in the light of new CO studies of SNRs, whether EGRET could have detected SNRs lying farther away than $5 \mathrm{kpc}$ from Earth.

\section{EGRET SOURCE - SUPERNOVA REMNANT PAIRS}

Table 1 shows those 3EG sources that are spatially coincident with SNRs listed in the latest version of Green's Catalog (2000). From left to right, columns are for the $\gamma$-ray source name, the measured flux in the summed EGRET phases P1234 (in units of $10^{-8} \mathrm{ph} \mathrm{cm}^{-2} \mathrm{~s}^{-1}$ ), the photon spectral index $\Gamma$, the EGRET class of source (em for possibly extended and $\mathrm{C}$ for confused), the variability indices $I$ (as in Torres et

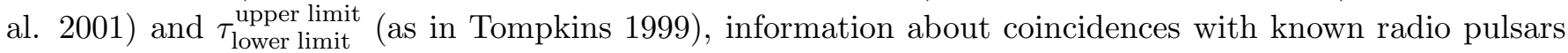
("y" stands for a known pulsar within the error box), the SNR identification (including other usual names when available), the angular distance between the center of the $\gamma$-ray source position and the center of the remnant (in degrees), the size of the remnant (in arcmin), and the SNR type T ( $\mathrm{S}$ for shell-like emission, $\mathrm{F}$ for filled-centre or plerionic remnant, and $\mathrm{C}$ for composite). The Poisson probability for the 19 coincidences shown in that table to be a chance effect (computed using thousands of simulated sets of EGRET sources, by means of a numerical code described elsewhere, Sigl et al. 2001) is less than $1.05 \times 10^{-5}$. Table 1 presents as well other features of the SNRs in the coincident pairs. Distances are only approximate since several different values for the same SNR can be found in the literature. When no direct determination is available, estimates can be made using the radio surface brightness-to-diameter relationship, known as $\Sigma-D$ (e.g. Case \& Bhattacharya 1998).

Using the estimated distance to each remnant in Table 1, we have calculated the approximate intrinsic $\gamma$-ray luminosity (in the energy range $100 \mathrm{MeV}-10 \mathrm{GeV}$, using the observed EGRET flux and photon indices, assuming isotropic emission). Figure 1 shows the computed luminosities as a function of the photon spectral index and the distance to each of the SNRs involved in Table 1 Looking at the left panel of Figure 1 three different parts of that plot appear to be distinguishable. First, at the bottom right, we find two sources presenting the highest spectral indices together with the highest levels of variability. It is unlikely that these two sources are related with the involved SNRs. Three sources, at the upper left corner, also appear, $a$ priori, to be unlikely related with the SNR: a tentative blazar classification has been made for one of them 
Table 1: Positional coincidences between supernova remnants quoted in Green's Catalog (2000), and unidentified 3EG EGRET sources. See text for the meaning of the different columns. The distance $d$ is in kpc. $F_{\text {radio }}^{1 \mathrm{GHz}}$ is in Jy, and $L_{\gamma}$ is in $\operatorname{erg~s}^{-1}$. The distances mentioned are reported or discussed in the references quoted below. Radio fluxes and spectral indices ( $\alpha$, such that $\left.S_{\nu} \propto \nu^{\alpha}\right)$ are taken from Green's (2000) Catalog.

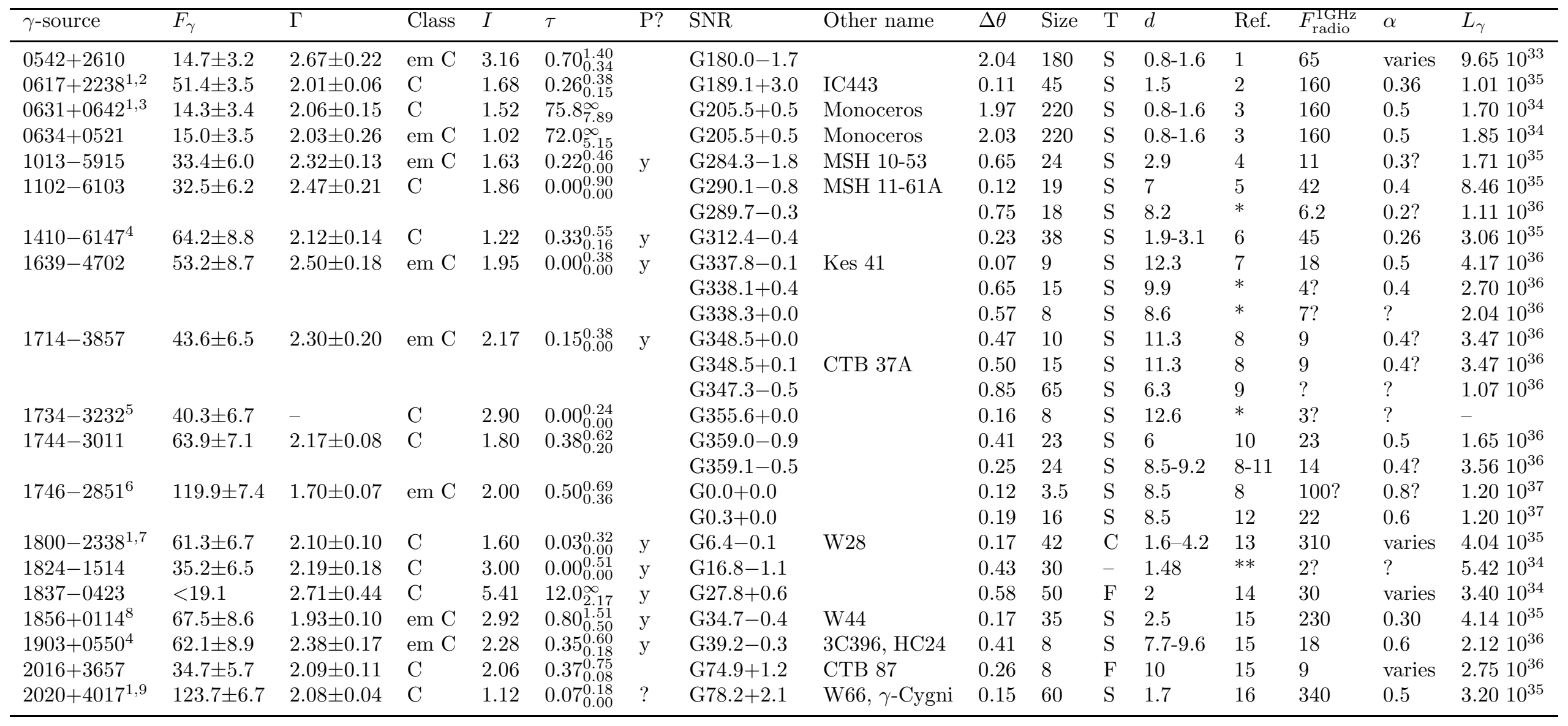

${ }^{1}$ Association proposed by Sturner \& Dermer (1995) and Esposito et al. (1996). ${ }^{2}$ GeV J0617+2237 ${ }^{3}$ GeV J0633+0645. ${ }^{4}$ Association proposed by Sturner \& Dermer (1995). ${ }^{5} \mathrm{GeV}$ J1732-3130. ${ }^{6} \mathrm{GeV}$ J1746-2854. ${ }^{7} \mathrm{GeV}$ J1800-2328. ${ }^{8} \mathrm{GeV}$ J1856-0115. ${ }^{9} \mathrm{GeV}$ J2020+4023. GeV sources compiled in the GeV ASCA Catalog (Roberts et al. 2001a). References quoted for the SNR distance (Column Ref.) are 1. Anderson et al. (1996) 2. Fesen (1984) 3. Jaffe et al. (1997) and Hensberge et al. (2000) 4. Ruiz \& May (1986) 5. Kaspi et al. (1997) 6. Caswell \& Barnes (1985), Case \& Bhattacharya (1999) 7. Koralesky et al. (1998) 8. Green et al. (1997), see also Reynoso \& Mangum (2000) 9. Slane et al. (1999) 10. Bamba et al. (2000) 11. Uchida, Morris \& Yusef-Zadeh (1992) 12. Kassim \& Frail (1996) 13. Frail et al. (1993) and Clark \& Caswell (1976) 14. Reich, Furst \& Sofue (1984) 15. Green (2000) and Caswell et al. (1975) 16. Lozinskaya et al. (2000)

* From the $\Sigma-D$ relationship presented by Case \& Bhattacharya (1998) ** Distance assumed equal to a coincident OB association, Romero, Benaglia \& Torres (1999). Please see Torres et al. (2002) for full bibliographic details. 

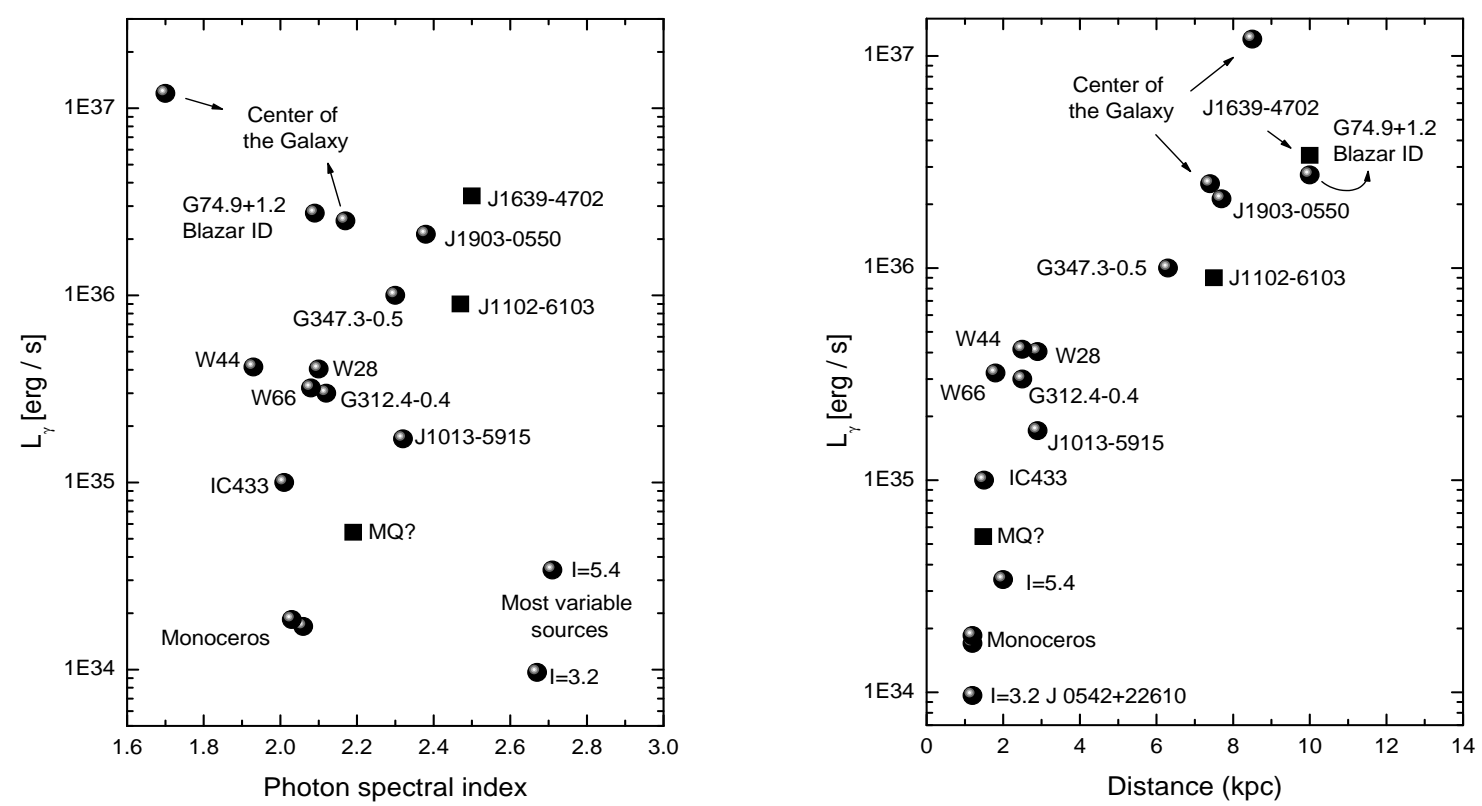

Fig. 1. Computed luminosities (see text) as a function of the photon spectral index and distances. Circles stand for SNRs whose distances has been determined, whereas squares stand for those which distances have been estimated using the $\Sigma-D$ relationship.

(Halpern et al. 2001a), whereas for the others, a high luminosity at too low spectral index would be required for Fermi's mechanism to be operative. Confusion related to their close position towards the center of the galaxy would make any identification even more dubious. The third region in the plot, the central one, which apparently shows a tendency to increase the required luminosity when increasing the photon spectral index, encompasses the rest of the sources. All the likely a priori candidates studied in the literature before lie there, including the well studied W44, IC433, W28, and W66.

\section{DISTANT SNRS AS GAMMA-RAY SOURCES?}

Disregarding the EGRET sources spatially close to, maybe even physically associated with, the galactic center, there are 5 cases in Table 1 which coincide with SNRs apparently located farther away than 5 kpc. Of these, 3EG J1714-3857, has been thoroughly studied in recent years (e.g. Slane et al. 1999, Butt et al. 2001, 2002, Muraishi et al. 2000). This is probably the most compelling case for acceleration of protons up to $\mathrm{TeV}$ energies in a SNR, with the bulk of the observed $\mathrm{GeV} \gamma$-rays being produced by proton-proton interaction and subsequent pion decays. The distance to the associated SNR, G347.3-0.5, is estimated to be $\sim 6 \mathrm{kpc}$ (Slane et al. 1999).

In the case of 3EG J1714-3857, we associated the unidentified EGRET GeV $\gamma$-ray source with a very massive $\left(\sim 3 \times 10^{5} M_{\odot}\right)$ and dense $\left(\sim 500\right.$ nucleons $\left.\mathrm{cm}^{-3}\right)$ molecular cloud interacting with the TeV $\gamma$-ray emitting, spatially coincident, SNR G347.3-0.5. Since the cloud region is of low radio and X-ray brightness, we were able to dismiss an electronic origin for the bulk of the GeV emission there. Furthermore, the ambient cloud medium is directly measured to be unusually excited, suggesting that it is indeed being overtaken by the SNR shock front. A picture then emerges where, even when the SNR lie farther than $\sim 5 \mathrm{kpc}$, it was possible for EGRET to detect it (Butt et al. 2001). A question then naturally arises: could EGRET have detected even farther SNRs?

\section{EG J1102-6103 - SNR G290.1-0.8 (MSH 11-61A)/289.7-0.3}

The line of sight to 3EG 1102-6103 intersects both the near and far sides of the Carina spiral arm, at velocities near $-20 \mathrm{~km} \mathrm{~s}^{-1}$ and $+20 \mathrm{~km} \mathrm{~s}^{-1}$ respectively. There is a distinct gap in the near side of the Carina arm in the direction of the 3EG source, with almost no molecular gas within $\sim 1$ deg of the source 
direction (see, e.g., Figure 2 of Dame, Hartmann, \& Thaddeus 2001). On the other hand, there is a very massive molecular complex in the far Carina Arm overlapping the direction of the 3EG source; this complex is No. 13 in the Carina Arm cloud catalog of Grabelsky et al. (1988). There is little doubt that the two component clouds, labeled A and B in Figure 14 of Torres et al. (2002) are part of the same complex, since they have approximately the same velocity of $22 \mathrm{~km} \mathrm{~s}^{-1}$, and are connected smoothly by weaker emission, also at the same velocity. Also, the HII regions are evidence of abundant on-going star formation in this molecular complex which additionally supports the association of the SNR. Assuming a flat rotation curve beyond the solar circle, the kinematic distance of the complex is $8.0 \mathrm{kpc}$ and its total molecular mass is 2.1 $\times 10^{6} M_{\odot}$.

It is worth noting that the composite CO line profile of cloud B is very broad and complex, suggesting possible interaction with SNR G290.1-0.8. In the case of cloud A, its radius ( 48 pc) and composite linewidth $\left(17 \mathrm{~km} \mathrm{~s}^{-1} \mathrm{FWHM}\right)$ are roughly consistent with the radius-linewidth relation found for large molecular complexes by Dame et al. (1986). For cloud B, however, its linewidth $\left(\sim 27 \mathrm{~km} \mathrm{~s}^{-1}\right)$ is about a factor of 3 too large compared to its radius $(\sim 28 \mathrm{pc})$. The coinciding SNR G289.7-0.3 is far from Cloud B, in a region of low molecular density. It is extremely unlikely that this SNR is related with the 3EG source in question. The only remaining candidate is, then G290.1-0.8. The total molecular mass within the 95\% confidence radius of the $3 \mathrm{EG}$ source is $7.7 \times 10^{5} M_{\odot}$ and most of it is localized in Cloud B $\left(4.5 \times 10^{5} M_{\odot}\right)$.

Assuming typical values for the energy of the explosion $\left(E_{51}=1\right)$ and the unshocked ambient density $\left(n=0.1 \mathrm{~cm}^{-3}\right)$ we obtain a $\mathrm{CR}$ enhancement factor of $\sim 250$. Assuming that the same CR enhancement is applicable to the cloud overpredicts the EGRET flux by about a factor of 10. Even with an average CR enhancement factor within the cloud ten times lower than within the SNR, it is at least possible, then, that 3EG J1102-6103 and SNR G290.1-0.8 are indeed related. Note that Bremsstrahlung, which we have neglected, will contribute still more to the predicted flux from SNR-cloud interactions. If the outlined scenario is correct, GLAST and AGILE will observe a strong, compact $\gamma$-ray source coincident with the position of Cloud B.

\section{EG J1639-4702 - SNR G337.8-0.1/338.1+0.4/338.3+0.0}

Based on HI absorption seen all the way up to the terminal velocity, Caswell et al. (1975) placed the SNR G337.8-0.1 beyond the tangent point at $7.9 \mathrm{kpc}$. Koralesky et al. (1998) detected maser emission in the SNR at $-45 \mathrm{~km} \mathrm{~s}^{-1}$, implying a far kinematic distance of $12.4 \mathrm{kpc}$. As Figure 17 of Torres et al. (2002) shows, there is a very massive giant molecular cloud adjacent to the SNR in direction and close to the associated maser in velocity $\left(-56 \mathrm{~km} \mathrm{~s}^{-1}\right)$. The far kinematic distance for this giant molecular cloud is favored by (1) its likely association with both the far-side maser just mentioned and a group of far-side HII regions (group 5 in Georgelin \& Georgelin 1976); (2) its location very close to the Galactic plane; and (3) the radius linewidth relation for giant molecular clouds (Dame et al. 1986). The mean velocity of the complex is $-56 \mathrm{~km} \mathrm{~s}^{-1}$, implying a far kinematic distance of $11.8 \mathrm{kpc}$.

Taking the total CO luminosity of the giant molecular cloud to be that in the range $l=337.625$ to 338.25, $b=-0.25$ to 0.25 , and $v=-65$ to $-45 \mathrm{~km} \mathrm{~s}^{-1}$, the total molecular mass is $5 \times 10^{6} M_{\odot}$; this mass may be overestimated by 10-20\% owing to the inclusion of emission from gas at the same velocity at the near kinematic distance. Even with this correction, this giant molecular cloud ranks among the few most massive in the Galaxy (see, e.g., Dame et al. 1986); its composite CO linewidth of $\sim 20 \mathrm{~km} \mathrm{~s}^{-1}$ is correspondingly very large. Adopting a mean radius of $0.31 \mathrm{deg}$, or $65 \mathrm{pc}$ at $11.8 \mathrm{kpc}$, the mean nucleon density of the cloud is $176 \mathrm{~cm}^{-3}$. The total mass within the $95 \%$ confidence radius of the $3 \mathrm{EG}$ source is $7.6 \times 10^{6} M_{\odot}$; this mass too may be overestimated by $10-20 \%$ owing to inclusion of near-side emission.

Again, there is so much molecular material that a conservative enhancement factor would be enough to produce much of the GeV flux detected by EGRET. It is at least possible that 3EG J1639-4702 is partially related to the SNRs with which it coincides. In addition, the Parkes pulsar PSR J1637-4642 seems to be a plausible candidate for the origin of this 3EG source as well: Only a $12 \%$ efficiency would be needed (Torres, Butt, \& Camilo 2001). Although the spectral index of the $3 \mathrm{EG}$ source, $\Gamma=2.50 \pm 0.18$, seems quite soft in comparison with detected EGRET pulsars, the work of Halpern et al. (2001) suggests that a soft spectral index does not automatically rule out a pulsar origin of the $\gamma$-rays. 


\section{EG J1903+0550 - SNR G39.2-0.3}

Caswell et al. (1975) detected HI absorption all the way up to the terminal velocity towards the SNR G39.2-0.3, with almost continuous strong absorption between $60 \mathrm{~km} \mathrm{~s}^{-1}$ and the terminal velocity. They therefore concluded that the remnant was certainly beyond the tangent point, and most likely at the far distance corresponding to $60 \mathrm{~km} \mathrm{~s}^{-1}, \sim 9.6 \mathrm{kpc}$. Such a large distance is consistent with the high foreground hydrogen column inferred both by Becker \& Helfand (1987) based on $21 \mathrm{~cm}$ absorption measurements with the VLA, and by Harrus \& Slane (1999) based on ASCA observations. A distance of $9.6 \mathrm{kpc}$ would place the SNR in the far Sagittarius arm, where the remnant is nearly coincident with a massive molecular complex. The complex is $(40,59)$ in the catalog of Dame et al. (1986), who assigned the far kinematic distance based on 2 associated HII regions. The mass of this complex is estimated to be $2.1 \times 10^{6} M_{\odot}$. The mass within the $95 \%$ confidence radius of the 3EG source (dotted circle in Figure 26 of Torres et al. 2002) is even higher, $3.4 \times 10^{6} M_{\odot}$, because the radius also includes part of another molecular complex at higher longitude. If part of the mass contained in the molecular complex could serve as target material for the relativistic particles accelerated in the SNR shock, this 3EG detection could plausibly be produced by a combination of Bremsstrahlung and pion decay. With the mass quoted, a CR enhancement factor of less than 10 is all that is needed to produce the bulk of the observed $\gamma$-ray emission. However, it is clear that not all of the molecular mass can be illuminated by the SNR shock front. The SNR itself is less than 8 arcmin in size, while the $3 \mathrm{EG}$ source is $\sim 1 \mathrm{deg}$ in size. Only $0.1 \%$ of the molecular material need to serve as a target for the particles accelerated in G39.2-0.3 in order to produce the 3EG source.

\section{EG J2016+3657 - SNR G74.9+1.2 (CTB 87)}

Although it has been suggested that SNR G74.9+1.2 (CTB 87) may be interacting with molecular clouds (Huang et al. 1983, Huang and Thaddeus 1986), the coincident 3EG J2016+3657 source has been proposed as a counterpart of the blazar-like radio source G74.87+1.22 (B2013+370) (Halpern et al. 2001a, Mukherjee et al. 2000). B2013+370 is a compact, flat spectrum, 2 Jy radio source at $1 \mathrm{GHz}$. Its multiwavelength properties were compiled by Mukherjee et al. (2000), and since they resemble other blazars detected by EGRET, B2013+370 is an interesting possible counterpart for this 3EG source.

The Crab-like supernova remnant CTB 87 is located at more than $10 \mathrm{kpc}$ (Green 2000), seemingly disfavoring its shell interactions as the cause of the EGRET source. There are also WR stars in the field (Romero et al. 1999), which might produce $\gamma$-ray emission. This possibility remains to be analyzed. INTEGRAL observations would help in determining if there is $\gamma$-ray emission coming from the stars. Figure 27 of Torres et al. (2002) shows a CO map for the CTB 87 region. One clearly defined molecular cloud appears in the map. The mean velocity of the molecular cloud is $-57 \mathrm{~km} / \mathrm{s}$. Assuming a flat rotation curve beyond the solar circle, the cloud's kinematic distance is $10.4 \mathrm{kpc}$, similar to that of the SNR. The total molecular mass within the $95 \%$ confidence radius of the $3 \mathrm{EG}$ source is $1.7 \times 10^{5} M_{\odot}$. With such a high value for the molecular mass, there is still a chance that the hadronic or leptonic $\gamma$-ray emission may be contributing to 3EG J2016+3657.

\section{CONCLUDING REMARKS}

It is at least plausible that EGRET has detected distant (more than $6 \mathrm{kpc}$ ) SNRs. There are 5 coinciding pairs of 3EG sources and SNRs for which the latter apparently lie at such high values of distance (disregarding those related with SNRs spatially close to the galactic center). For all these cases, we have uncovered the existence of nearby, large, in some cases giant, molecular clouds that could enhance the GeV signal through pion decay. It is possible that the physical relationship between the 3EG source and the coinciding SNR could provide for these pairs a substantial part of the $\mathrm{GeV}$ emission observed. This does not preclude, however, composite origins for the total amount of the radiation detected. Some of these cases present other plausible scenarios (see Torres et al. 2002 for details). AGILE observations, in advance of GLAST, would greatly elucidate the origin for these $3 \mathrm{EG}$ sources, since even a factor of 2 improvement in resolution would be enough to favor or reject the SNR connection.

\section{ACKNOWLEDGEMENTS}

The work of DFT was performed under the auspices of the U.S. Department of Energy by University of California LLNL under contract No. W-7405-Eng-48. GER and JAC. were supported by CONICET (under 
grant PIP N ${ }^{o}$ 0430/98), ANPCT (PICT 03-04881), as well as by Fundación Antorchas. YMB acknowledges the support of the High Energy Astrophysics division at the CfA and the Chandra project through NASA contract NAS8-39073.

\section{REFERENCES}

Aharonian F.A., \& Atoyan, A.M., On the emissivity of $\pi^{0}$-decay gamma radiation in the vicinity of accelerators of galactic cosmic rays, 1996, A\&A 309, 91

Becker R.H., \& Helfand D.J., High-resolution X-ray and radio images of the galactic SNR G39.2-0.3, 1987, AJ 94, 1629

Butt Y., Torres D.F., Combi J.A., Dame T., \& Romero G.E., Is the supernova remnant RX J1713.7-3946 a hadronic cosmic ray accelerator ?, 2001, ApJ 562, L167

Butt Y., Torres D.F., Romero G.E., Dame T., \& Combi J.A., Supernova-remnant origin of cosmic rays?, 2002, Nature 418, 499

Case G., \& Bhattacharya D., A New Sigma -D Relation and Its Application to the Galactic Supernova Remnant Distribution, 1998, ApJ 504, 761

Caswell J.L., Murray J.D., Roger R.S., Cole D.J., \& Cooke D.J., Neutral hydrogen absorption measurements yielding kinematic distances for 42 continuum sources in the galactic plane, 1975, A\&A 45, 239

Dame T.M., Elmegreen B.G., Cohen R.S., \& Thaddeus P. 1986, The largest molecular cloud complexes in the first galactic quadrant, ApJ 305, 892

Dame T.M., Hartmann D., \& Thaddeus P., The Milky Way in Molecular Clouds: A New Complete CO Survey, 2001, ApJ 547, 792

Harrus I.M. \& Slane P.O., An ASCA Study of the Supernova Remnant G39.2-0.3, 1999, ApJ 516, 811

Halpern J.P., et al., PSR J2229+6114: Discovery of an Energetic Young Pulsar in the Error Box of the EGRET Source 3EG J2227+6122, 2001, ApJ 552, L125

Halpern J.P., Eracleous M., Mukherjee R., \& Gotthelf E.V., 3EG J2016+3657: Confirming an EGRET Blazar behind the Galactic Plane, 2001a, ApJ 551, 1016

Georgelin Y.P. \& Georgelin Y.M. 1976, The spiral structure of our Galaxy determined from H II regions, A\&A 49, 57

Grabelsky D.A., Cohen R.S., Bronfman L., \& Thaddeus P., Molecular clouds in the Carina arm - The largest objects, associated regions of star formation, and the Carina arm in the Galaxy 1988, ApJ 331, 181

Green D.A. 2000, A Catalogue of Galactic Supernova Remnants, Mullard Radio Astronomy Observatory, Cambridge, UK (available at http://www.mrao.cam.ac.uk/surveys/snrs/)

Koralesky B., Frail D.A., Goss W.M., Claussen M.J., \& Green A.J., Shock-excited Maser Emission from Supernova Remnants: G32.8-0.1, G337.8-0.1, G346.6-0.2, and the HB 3/W3 Complex 1998, AJ, 116, 1323

Mukherjee R., Gotthelf E.V., Halpern J., \& Tavani M.,Multiwavelength Examination of the COS B Field 2CG 075+00 Yields a Blazar Identification for 3EG J2016+3657 2000, ApJ 542, 740

Muraishi H., et al., Evidence for TeV gamma-ray emission from the shell type SNR RX J1713.7-3946, 2000, A\&A 354, 57L

Romero G.E., Benaglia P., \& Torres D.F.,Unidentified 3eg gamma-ray sources at low galactic latitude, 1999, A\&A 348, 868

Sigl G., Torres D.F, Anchordoqui L.A., \& Romero G.E., Testing the correlation of ultrahigh-energy cosmic rays with high redshift sources, 2001, Phys. Rev. D63, 081302

Slane P., et al. 1999, Nonthermal X-Ray Emission from the Shell-Type Supernova Remnant G347.3-0.5, ApJ 525, 357

Tompkins W., Applications of Likelihood Analysis in Gamma-Ray Astrophysics, 1999, Ph.D. Thesis, Stanford University.

Torres D.F., Butt Y.M. \& Camilo F., Recently discovered pulsars and unidentified egret sources, 2001, ApJ 560, L155

Torres D.F., et al., Low latitude gamma-ray sources and the hypothesis of a black hole population, 2001a, A\&A 370, 468

Torres D.F, Romero G.E., Dame T. M., Combi J.A., \& Butt Y.M., Supernova remnants and gamma-ray sources, 2002, astro-ph/0209565 